\title{
Terminology, criteria, and definitions in complex regional pain syndrome: challenges and solutions
}

\author{
This article was published in the following Dove Press journal: \\ Journal of Pain Research \\ II December 2015 \\ Number of times this article has been viewed
}

\author{
Katherine Dutton' \\ Geoffrey Littlejohn² \\ 'Department of Rheumatology, \\ Townsville Hospital, Douglas, QLD, \\ Australia; ${ }^{2}$ Department of Medicine, \\ Monash University, Melbourne, VIC, \\ Australia
}

Correspondence: Geoffrey Littlejohn Suite H, Monash Medical Centre, 246 Clayton Road, Clayton, Melbourne, VIC, Australia

Email geoff.littlejohn@monash.edu

\begin{abstract}
Complex regional pain syndrome has long been recognized as a severe and high impact chronic pain disorder. However, the condition has historically been difficult to define and classify and little attention has been given to where complex regional pain syndrome sits within other apparently similar chronic pain disorders, such as fibromyalgia and regional pain syndrome. In this review challenges in regard to nomenclature, definitions, and classification of complex regional pain syndrome are reviewed and suggestions are provided about future directions.
\end{abstract}

Keywords: complex regional pain syndromes, fibromyalgia, causalgia, classification

\section{Introduction}

Complex regional pain syndrome (CRPS) has remained one of the most enigmatic of disease entities since first described over 150 years ago. Primarily presenting as a chronic pain condition that usually affects a single limb, it is defined by a composite of characteristic symptoms and signs. ${ }^{1,2}$ The central feature is severe, often debilitating pain. This is accompanied by a collection of sensory, motor, autonomic, skin, and/or bone abnormalities. A key feature is allodynia, where otherwise innocuous stimulation will cause pain and hyperalgesia, where there is exaggerated pain to a known painful stimulus. The patient will present with varying degrees of pain, allodynia, hyperalgesia, swelling, and color and temperature change. There are often changes in motor function, such as muscle stiffness or even involuntary movements. Regional osteopenia, changes to hair and nail growth, and dystrophic cutaneous changes may occur. ${ }^{1-3}$ The magnitude of each of these features will vary between individuals, each existing on a spectrum. The resulting clinical phenotype is characteristic and defines the condition. CRPS has high impact in terms of individual, health care, and economic burden yet despite this continues to lack a clear biological explanation and predictable effective treatment. Despite its sizable disease burden, its long history of identification, and a concentrated research effort many significant challenges remain. These relate to issues of terminology, diagnostic criteria, pre-disposing factors, triggers, pathophysiology, and the ideal treatment path. There is also the challenge of the well-guarded notion that CRPS is not a true disease state, but rather a condition that is the result of psychological foundations. This review focuses on the challenges that exist in diagnosing and classifying CRPS and to consider tenable solutions. 


\section{Challenge I: recognition of the condition of CRPS and its terminology}

The term used to describe a disease or clinical condition is important as it provides not only a means for medical communication, but also an accurate framework for understanding the causal mechanisms, pathophysiology, and clinical characteristics of the condition. ${ }^{4}$ In the case of CRPS there have been many attempts to find a universally accepted name that suits this condition resulting in the existence of a wide variety of terms (Table 1). ${ }^{5,6}$ As many as 202 names, expressed in several languages, have been identified, the names reflecting various theories held related to the initializing event, pathogenic mechanisms, clinical expression or to the name of the author. ${ }^{4,7}$ Causalgia, from the Greek meaning heat and pain, was the founding term for the syndrome. This was first used by Mitchell et al to represent the burning nature of the pain, as seen within American Civil War casualties suffering traumatic bullet wounds. ${ }^{89}$ With the advent of radiology the clinical features of CRPS were greatly expanded. ${ }^{10}$ In the 1940s, when the hypothesis that the sympathetic nervous system played a key role in the development and maintenance of the disease, the term "reflex sympathetic dystrophy" was introduced. ${ }^{11}$ In an attempt to introduce scientific strategy to the disease concept, "causalgia" came to be used to identify the syndrome in the presence of a nerve lesion, while "reflex sympathetic dystrophy" was left to describe the syndrome without such evidence. However, this latter term was found to be too simplistic and has been discarded. For instance, it has been noted that only $15 \%$ of patients develop dystrophy and the role of a simple peripheral reflex being driven by the sympathetic nervous system has not been validated. ${ }^{2}$

In 1994, a working group for the International Association for the Study of Pain (IASP) held a consensus conference to develop a more neutral term in recognition of the need to address the widespread inconsistency in terminology and to avoid reference to unsubstantiated theory on causation and

Table I Selected nomenclature used for complex regional pain syndrome 6

\begin{tabular}{ll}
\hline Causalgia & Algodystrophy \\
Sudeck's atrophy & \\
Reflex (sympathetic) dystrophy & Post-traumatic osteoporosis \\
syndrome & \\
Shoulder hand syndrome & \\
Transient osteoporosis & Regional migratory osteoporosis \\
\hline
\end{tabular}

Note: Copyright (O) 1998. Littlejohn GO. Algodystrophy (reflex sympathetic dystrophy syndrome). In: Maddison PJ, Isenberg DA, Woo P, Glass DN, editors. Oxford Textbook of Rheumatology.Vol 2. Oxford: Oxford University Press; 1998: 1679-1689.6 Reproduced by permission of Oxford University Press www.oup.com. etiology. ${ }^{12}$ Born out of this meeting came the "officiallyendorsed" term "complex regional pain syndrome" a denomination intended to be descriptive, general, and not to imply any etiopathology. ${ }^{13}$ The term was further sub-differentiated into "CRPS 1" if it occurred in the presence of a nerve injury (thus replacing the term causalgia) and "CRPS 2" if without evidence of nerve injury (replacing the term reflex sympathetic dystrophy). Since its introduction, there has been increasing acceptance of this term. A 2013 review by Todorova et al reported that $79 \%$ of all articles published on the topic since 2001 were using the new name with evidence of a marked gradual increase in uptake of its use between subsequent years. ${ }^{4}$

Although the evidence suggests that the new terminology has been met with overall acceptance, this has not been without pools of resistance. Firstly, there has been criticism over the nomination of an "umbrella term" citing that is too vague and lacking in historical reference. ${ }^{7}$ Secondly, given that the syndrome can in some cases affect the contralateral or ipsilateral extremity it is inappropriate to lend nominal reference to the idea that the syndrome is "regional". ${ }^{14}$

The differentiation of CRPS into subtypes 1 and 2 based on presence of a nerve injury has also been disputed. ${ }^{15}$ In a study by Harden et al of CRPS type 1 and type 2, as determined by nerve conduction studies, there was no difference found in the frequency of any clinical parameter between the two subtypes. ${ }^{16}$ While routine nerve conduction studies only define dysfunction in the large and not the small peripheral nerves, this study has demonstrated that clinical distinction between the subtypes cannot be substantiated on scientific grounds. It has also been suggested that CRPS type 1 and CRPS type 2 are both associated with nerve injury and that type 1 may represent a small fiber predominant mono- or oligoneuropathy that is initiated by limb trauma. ${ }^{17-19}$

\section{Comment}

The current terminology represents a compromise and remains a work in progress. It will likely undergo modifications in the future as specific mechanisms of causation are better defined. It is essential that any future denomination of the syndrome will facilitate research and treatment.

\section{Challenge 2: establishment of diagnostic criteria}

In the past, CRPS was diagnosed using a variety of nonstandardized and idiosyncratic diagnostic systems, each of which was derived solely from the authors' clinical experience and none of which achieved wide acceptance., ${ }^{2,20}$ 
The initial IASP criteria provided the ground rules for clinical diagnosis and aided research. ${ }^{13}$

However, the diagnostic accuracy of the criteria was later improved by more complete grouping of clinical symptoms and signs. ${ }^{21}$ These subsequent iterations of criteria for CRPS are known as the Budapest criteria (Tables 2 and 3 ). They comprise a criteria set for clinical diagnosis (sensitivity 0.85 , specificity 0.69 ) and a more stringent set for research (sensitivity 0.75 and specificity 0.96). ${ }^{22}$ It is suggested that these criteria present a reasonable compromise between sensitivity and specificity in the clinical setting. However, it has been cautioned that too high sensitivity may lead to "over diagnosis" of CRPS and the use of potentially harmful treatments when these may not be needed. ${ }^{2}$ The clinician needs to judge when pain is "disproportionate in time or degree to the usual course of pain after any trauma or other inciting event". Different traumas or events may have different healing times, and deviation from that should alert the

Table 2 "Budapest" clinical diagnostic criteria for complex regional pain syndrome ${ }^{22}$

Each of the following criteria must be met:

I. Patients must report continuing pain that is disproportionate in time or degree to the usual course of pain after any trauma or other inciting event.

2. Patients must report at least one symptom in three of the four following categories:

a. sensory: hyperalgesia, (that is, exaggerated pain to a painful stimulus, such as pinprick) and/or allodynia (that is, pain elicited by a normally non-painful stimulus, such as light touch).

b. Vasomotor: skin color changes and/or skin color asymmetry and/ or temperature asymmetry.

c. Sudomotor/edema: edema and/or sweating changes and/sweating asymmetry.

d. Motor/trophic: decreased range of motion and/or motor dysfunction (weakness, tremor, dystonia) and/or trophic changes/ asymmetry involving nails, skin, and/or hair.

3. Patients must display at least one sign at the time of assessment in two (clinical) or three or more (research) of the following categories:

a. Sensory: hyperalgesia, (that is, exaggerated pain to a painful stimulus, such as pinprick) and/or allodynia (that is, pain elicited by a normally non-painful stimulus, such as light touch and/or deep somatic pressure and/or joint movement).

b. Vasomotor: temperature asymmetry and/or skin color changes and/or asymmetry.

c. Sudomotor/edema: edema and/or sweating changes and/or sweating asymmetry.

d. Motor/trophic: decreased range of motion and/or motor dysfunction (weakness, tremor, dystonia) and/or trophic changes involving nails, skin, and/or hair.

4. Signs and symptoms must not be better explained by another diagnosis

Note: Reprinted with permission from Harden RN, Bruehl S, Perez RS, et al.Validation of proposed diagnostic criteria (the "Budapest Criteria") for Complex Regional Pain Syndrome. Pain. 2010;150(2):268-274.22 Promotional and commercial use of the material in print, digital or mobile device format is prohibited without the permission from the publisher Wolters Kluwer Heath. Please contact healthpermissions@ wolterskluwer.com for further information. clinician to the possibility to CRPS. ${ }^{23,24}$ This judgment is not always easy and it is common for the treating clinician to delay diagnosis. Additionally, the clinical features of early CRPS are similar to those of normal healing, depending on the trauma and the part involved. ${ }^{25}$ In fact, the processes involved in CRPS can be seen as an exaggerated form of those same processes involved in normal healing. These resolve in a timely fashion in normal healing while the same processes continue and amplify in CRPS. Clinical clues for high risk of development of CRPS in this context include, for example, a high level of pain $(>5 / 10) 1$ week after wrist trauma. ${ }^{26}$

The Budapest criteria thus provide a solid diagnosis for established and severe CRPS. They enhance clinical diagnosis and research into more severe CRPS. However, CRPS does not exist as a black and white phenomenon, being present just when the criteria are met and not being present when they just fall short. This is partly recognized by the additional subtype of CRPS, termed "CRPS not otherwise specified", to include patients who do not reach the new clinical criteria but whose clinical features appear to fall within the CRPS paradigm and who have no other identifiable explanation for their condition. ${ }^{22}$

Like many pain syndromes CRPS is a spectrum disorder. The clinical features vary in intensity and in their clinical presentation. Thus a scale is needed that captures the essential elements of the disorder and rates where an individual will sit on the scale and also if a diagnostic threshold is passed within that scale. Each individual with persisting regional pain could then be assessed in a standardized way. This would assist in awareness of the possibility of CRPS and early diagnosis thus accelerating focused management strategies.

This approach has been found to be very useful in fibromyalgia, a condition typically characterized by wide-spread pain rather than regional pain, but with many features that are seen in CRPS. The Polysymptomatic Distress Score (called by some the Central Sensitivity Score) ${ }^{27}$ is derived from a compilation of the number of painful or tender pre-specified regions that a patient has plus a rating of the severity of sleep disturbance, levels of fatigue and cognitive dysfunction, as well as the presence of headache, abdominal pain, and depression. ${ }^{28,29}$ This score can be used to diagnose fibromyalgia according to whether or not the patient crosses a certain pre-defined and validated threshold score. The score also identifies the tendency of any individual to have central sensitivity. ${ }^{27}$ Those with low scores have less tendency to central sensitivity and those with higher scores have more. For instance, this score has been shown to predict poorer post-operative pain outcomes. ${ }^{30} \mathrm{CRPS}$ would lend itself to a similar diagnostic and rating strategy. 
Table 3 Comparison of selected clinical features in different definitions of complex regional pain syndrome (CRPS)

\begin{tabular}{|c|c|c|c|c|}
\hline CRPS terminology & $\begin{array}{l}\text { CRPS type I } \\
\text { Orlando } 1994\end{array}$ & $\begin{array}{l}\text { CRPS type } 2 \\
\text { Orlando } 1994\end{array}$ & $\begin{array}{l}\text { CRPS } \\
\text { Bruehl } 1999\end{array}$ & $\begin{array}{l}\text { CRPS } \\
\text { Budapest } 2003\end{array}$ \\
\hline Physical trigger (nerve in type 2 ) & \pm & $\checkmark$ & & \\
\hline Disproportionate pain, allodynia or hyperalgesia & $\checkmark$ & $\checkmark$ & & \\
\hline Disproportionate pain & & & $\checkmark$ & $\checkmark$ \\
\hline Signs: swelling/vasomotor/sudomotor & $\checkmark$ & $\checkmark$ & & \\
\hline $\begin{array}{l}\text { Symptoms: sensory/vasomotor/sudomotor/ } \\
\text { edema/motor/trophic }\end{array}$ & & & 4 of 4 & $\geq 3$ of 4 \\
\hline Signs: sensory/vasomotor/sudomotor/edema & & & $\geq \mathrm{I}$ in $\geq 2$ & $\geq 2$ of 4 \\
\hline Exclude explanatory pathology & $\checkmark$ & $\checkmark$ & $\checkmark$ & $\checkmark$ \\
\hline Sensitivity & 0.99 & & & 0.85 \\
\hline Specificity & 0.68 & & & 0.69 \\
\hline
\end{tabular}

Notes: $\checkmark=$ present; $\pm=$ may or may not be present.

The issues with diagnosis in CRPS are highlighted in a study of CRPS developing after limb trauma. ${ }^{23}$ Of 596 patients, only $7 \%$ were diagnosed with the current IASP criteria (Table 2), 49\% were diagnosed by previous 1994 criteria, and $21 \%$ with an alternative set of criteria. ${ }^{31}$

\section{Comment}

Current diagnostic criteria are very rigid and define patients with more severe or established CRPS, both in the clinic and for research purposes. Patients with lesser or earlier CRPS are not captured by these criteria yet early diagnosis and treatment seem the logical way to progress in order to achieve better outcomes. New criteria acknowledging CRPS as a spectrum diagnosis are needed.

\section{Challenge 3: defining subtypes of CRPS}

Just as there is controversy regarding subtyping CRPS into types 1 and 2, based on the presence or absence of significant neural damage as described above, so is there little consensus on staging CRPS according to duration or severity. At one time CRPS was empirically staged according to specific characteristics that occurred in a suggested time-frame (Table 4). ${ }^{32,33}$ This staging provided at best only very limited prognostic information on a small group of patients with very severe CRPS and has not been found to be clinically useful in the majority. Other suggested ways of subtyping CRPS have been examined but not achieved universal acceptance. ${ }^{34}$ The CRPS severity score provides grading of severity of CRPS but cutoff scores for various outcome measures have not yet been established. ${ }^{35}$

In clinical practice it is hard to predict the outcome at first presentation in any one patient with CRPS. It is also hard to predict response to different treatment interventions and hence to predict overall prognosis in CRPS. Certain age groups, such as adolescents, seem to have better and more positive predictive outcomes than others. ${ }^{36}$ In general, early diagnosis and early intervention with a variety of strategies, including education, psychological support, and mobilization, remain a hallmark of standard care. Additionally, early intervention with evidence-based treatments will improve outcomes across a range of clinical presentations. ${ }^{37}$

\section{Comment}

Better classification of subtypes of CRPS may aid choice of management strategies and clinical outcomes.

\section{Challenge 4: relationship of CRPS to other common pain syndromes}

Regional pain syndrome denotes a syndrome characterized by pain, tenderness, and other sensory symptoms, such as numbness and dysesthesia, which are present in a localized region. ${ }^{38}$ This is usually in a segmental distribution linked to the spine often giving rise to common presentations that are given descriptive and often inappropriate names. These include repetitive strain syndrome, whiplash, and low back pain syndrome. ${ }^{39}$ Regional pain syndrome shares many clinical features with CRPS, as denoted in Table 5, however these syndromes always have clinical features, such as pain, stiffness or tenderness that are present proximally. Both conditions are triggered by a previous, usually physical, event. Both need careful clinical assessment to exclude a condition that

Table 4 Previous staging criteria for severe complex regional pain syndrome

\begin{tabular}{lll}
\hline Stage & Characteristics & Time-frame \\
\hline I & $\begin{array}{l}\text { Pain, tenderness, swelling, vasomotor, } \\
\text { sudomotor changes }\end{array}$ & Acute \\
2 & Dystrophic changes & \\
3 & Atrophic changes & Several months \\
\end{tabular}


could cause mimicking symptoms. This is often a problem in regional pain syndrome where imaging of the spine will often show constitutional anatomical changes that may be attributed to the presenting clinical features.

Another feature linking these two conditions is the inevitable presence of variable levels of pain, tenderness or stiffness in the spinal region on the side of the CRPS. For instance, patients with CRPS affecting the wrist/hand region will also have neck and shoulder girdle signs on the affected side.

Thus both can have variable shared clinical features, such as regional pain, allodynia, and sensory symptoms. In CRPS these are more severe and peripheral but the two conditions are deemed to be on the same spectrum. The more pronounced and more easily defined clinical features of CRPS allow for better validated diagnostic criteria compared to regional pain syndrome. ${ }^{38}$

An additional link between CRPS and other musculoskeletal pain syndromes derives from the 2010 American College of Rheumatology diagnostic criteria for fibromyalgia. ${ }^{40}$ These criteria were designed to be more clinically useful for fibromyalgia diagnosis compared to the existing 1990 American College of Rheumatology classification criteria. This occurred through the elimination of the need for a certain number of tender points to be present on examination. The tender point requirement was replaced by the number of self-reported painful or tender areas plus a high level of common symptoms, namely sleep disturbance, fatigue, and cognitive problems, as well as adding in the presence of headache, abdominal pain or depression. Fibromyalgia is diagnosed if there are seven or more painful regions and high level of symptoms, or if there are three to six painful regions plus very high levels of the previously mentioned symptoms. Thus these latter criteria for fibromyalgia will include most patients with regional pain syndrome, as these patients have regional pain affecting at least three regions and usually high scores of the designated symptoms. Thus the spectrum of symptoms in CRPS extends to fibromyalgia (Table 6).

There are common mechanisms involved in fibromyalgia and CRPS. These include a number of key neurophysiological mechanisms. The first is that of neuro-inflammation through release of inflammatory mediators predominantly from C-type fibers in the periphery. ${ }^{41,42}$ These mediators include calcitonin gene related peptide, substance $\mathrm{P}$, and neurokinin A, among others. These neuropeptides induce regional inflammation comprising vasodilatation, vascular permeability with regional edema, and egress of polymorphonuclear leukocytes. These substances also act to sensitize peripheral pain nerves thus enhancing allodynia. Additional effects involve the innate immune cells and adaptive immune cells. Neuro-inflammation is a key feature of both disorders but seems more intense in a regionalized fashion in CRPS. In both conditions there is significant abnormality in peripheral nociceptive nerve anatomy, particularly the C-fibers. There is decreased density of such fibers in both disorders. ${ }^{3,43,44}$

Both disorders also exhibit evidence of central sensitization and both disorders show significant changes in central pain-related regions of the brain., ${ }^{3,45}$ The parallels between fibromyalgia and CRPS lead one to indicate that the two disorders exist on the same spectrum. Improved understanding of the pathophysiology of CRPS will allow for better classification of CRPS. ${ }^{3}$

\section{Comment}

The literature on fibromyalgia, regional pain syndrome, and CRPS tend to ignore contributions and observations from each of the other entities. Divide and conquer in this setting does not work. Understanding and incorporation of knowledge from each of these clinically-defined disorders is likely to enhance classification of the whole group and hence improve understanding of these important and high impact chronic pain disorders.

Table 5 Characteristics of complex regional pain syndrome (CRPS) compared to regional pain syndrome

\begin{tabular}{|c|c|c|c|}
\hline & $\begin{array}{l}\text { CRPS type I } \\
\text { IASP I994' }\end{array}$ & $\begin{array}{l}\text { CRPS Budapest } \\
2003^{22}\end{array}$ & $\begin{array}{l}\text { Regional pain } \\
\text { syndrome }\end{array}$ \\
\hline Physical trigger (nerve in type 2) & \pm & & \pm \\
\hline Disproportionate pain, allodynia or hyperalgesia & $\checkmark$ & & \\
\hline Disproportionate pain & & $\checkmark$ & $\checkmark$ \\
\hline Signs: swelling/vasomotor/sudomotor & $\checkmark$ & & \\
\hline Symptoms: sensory/vasomotor/sudomotor/edema/motor/trophic & & $\geq 3$ of 4 & \pm \\
\hline Signs: sensory/vasomotor/sudomotor/edema & & $\geq 2$ of 4 & \pm \\
\hline Exclude explanatory pathology & $\checkmark$ & $\checkmark$ & $\checkmark$ \\
\hline
\end{tabular}

Notes: $\checkmark=$ present; $\pm=$ may or may not be present.

Abbreviation: IASP, International Association for the Study of Pain. 
Table 6 Comparative clinical features of complex regional pain syndrome (CRPS), regional pain syndrome, and fibromyalgia

\begin{tabular}{llll}
\hline & CRPS & Regional pain Fibromyalgia \\
& Budapest 2003 $^{22}$ & syndrome & \\
\hline Pain distribution & Regional & Regional & Wide-spread \\
Sensory & $\checkmark \checkmark$ & $\checkmark$ & $\checkmark$ \\
Vascular & $\checkmark \checkmark$ & $\checkmark$ & $\checkmark$ \\
Edema/sweating & $\checkmark \checkmark$ & $\checkmark$ & $\checkmark$ \\
Motor/trophic & $\checkmark \checkmark$ & $\checkmark$ & $\checkmark$ \\
Emotional distress & $\checkmark$ & $\checkmark$ & $\checkmark$ \\
Exclude explanatory & $\checkmark$ & $\checkmark$ & $\checkmark$ \\
pathology & & & \\
\hline
\end{tabular}

Notes: $\checkmark=$ present, $\checkmark \checkmark=$ present and more marked.

\section{Summary}

Challenges exist in the terminology, definitions, and classification of CRPS. The progress made so far needs to be expanded through appreciation of the clinical features and pathophysiology of other similar and related chronic pain disorders. We suggest that better understanding and documentation of CRPS as a spectrum disorder rather than a dichotomous disorder will allow for earlier diagnosis and interventions with the likelihood of better prognosis.

\section{Disclosure}

The authors have no conflict of interest to disclose.

\section{References}

1. Birklein F, O'Neill D, Schlereth T. Complex regional pain syndrome: An optimistic perspective. Neurology. 2015;84(1):89-96.

2. Huygen F, O'Connell N, Harden N. Complex regional pain syndrome: state of the art. In: Raja SN, Sommer CL, editors. Pain 2014 Refresher Courses, 15th World Congress on Pain. Washington: IASP Press; 2014:259-272.

3. Birklein F, Schlereth T. Complex regional pain syndrome-significant progress in understanding. Pain. 2015;156 Suppl 1:S94-S103.

4. Todorova J, Dantchev N, Petrova G. Complex regional pain syndrome acceptance and the alternative denominations in the medical literature. Med Princ Pract. 2013;22(3);295-300.

5. Rhiannon J. Complex regional Pain Syndrome. In: West S, editor. Rheumatology Secrets. Third ed. Philadelphia: Elsevier; 2015.

6. Littlejohn GO. Algodystrophy (reflex sympathetic dystrophy syndrome). In: Maddison PJ, Isenberg DA, Woo P, Glass DN, editors. Oxford Textbook of Rheumatology. Vol 2. Oxford: Oxford University Press; 1998:1679-1689.

7. Alverez-Lario B, Arexabala-Alcibar I, Alegre-Lopez J, AlonsoValdivielso JL. Acceptance of the different denominations for Reflex Sympathetic Dystrophy. Ann Rheum Dis. 2001;60(1):77-79.

8. Mitchell SW, Morehouse GR, Keen WW. Gunshot Wounds and Other Injuries of Nerves. Philadelphia: Lippincott; 1864.

9. Lau FH, Chung KC. Silas Weir Mitchell, MD: the physician who discovered causalgia. J Hand Surg Am. 2004;29(2):181-187.

10. Sudeck P. Ueber die acute entzundliche knochenatrophie. [Concerning the acute inflammatory bone atrophy]. Arch Klin Chir. 1900;62:147156. German.

11. Evans JA. Reflex sympathetic dystrophy. Surg Clin North Am. 1946;26: 780-790.
12. Stanton-Hicks M, Janig W, Hassenbusch S, Haddox JD, Boas R, Wilson P. Reflex sympathetic dystrophy: changing concepts and taxonomy. Pain. 1995;63(1):127-133.

13. Merskey H, Bogduk N. Classification of Chronic Pain: Description of Chronic Pain Syndromes and Definitions of Pain Terms. Seattle: IASP Press; 1994.

14. Van Rijn MA, Marinus J, Putter H, Bosselaar SR, Moseley GL, van Hilten JJ. Spreading of complex regional pain syndrome: not a random process. J Neural Transm. 2011;118(9):1301-1309.

15. Schott GD. Complex? Regional? Pain? Syndrome? Pract Neurol.2007; 7(3):145-157.

16. Harden RN, Bruehl S, Galer BS, et al. Complex regional pain syndrome: are the IASP diagnostic criteria valid and sufficiently comprehensive? Pain. 1999;83(2):211-219.

17. Oaklander AL, Rismiller JG, Gelman LB, Zheng L, Chang Y, Gott R. Evidence of focal small-fiber axonal degeneration in complex regional pain syndrome-1(reflex dystrophy syndrome). Pain. 2006;120(3): 235-243.

18. Oaklander ALFH. Is reflex sympathetic dystrophy/complex regional pain syndrome type 1 a small-fiber neuropathy? Ann Neurol. 2009; 65(6):629-638.

19. Albrecht PJ, Hines S, Eisenberg E, et al. Pathologic alterations of cutaneous innervation and vasculature in affected limbs from patients with complex regional pain syndrome. Pain. 2006;120(3):244-266.

20. Bonica JJ. The Management of Pain. Philadelphia: Lea and Feibiger; 1953.

21. Bruehl S, Harden RN, Galer BS, et al. External validation of IASP diagnostic criteria for Complex Regional Pain Syndrome and proposed research diagnostic criteria. International Association for the Study of Pain. Pain. 1999;81(1-2):147-154.

22. Harden RN, Bruehl S, Perez RS, et al. Validation of proposed diagnostic criteria (the "Budapest Criteria") for Complex Regional Pain Syndrome. Pain. 2010;150(2):268-274.

23. Beerthuizen A, Stronks DL, Van't Spijker A, et al. Demographic and medical parameters in the development of complex regional pain syndrome type 1 (CRPS1): prospective study on 596 patients with a fracture. Pain. 2012;153(6):1187-1192.

24. Zollinger PE, Tuinebreijer WE, Breederveld RS, Kreis RW. Can vitamin $\mathrm{C}$ prevent complex regional pain syndrome in patients with wrist fractures? A randomized, controlled, multicenter dose-response study. J Bone Joint Surg Am. 2007;89(7):1424-1431.

25. Pepper A, Li W, Kingery WS, Angst MS, Curtin CM, Clark JD. Changes resembling complex regional pain syndrome following surgery and immobilization. J Pain. 2013;14(5):516-524.

26. Moseley GL, Herbert RD, Parsons T, et al. Intense pain soon after wrist fracture strongly predicts who will develop complex regional pain syndrome: prospective cohort study. J Pain. 2014;15(1):16-23.

27. Littlejohn G, Guymer E. Rename fibromyalgia score to Central Sensitivity Score to reflect what it measures. Arthritis Rheumatol. 2015;67(9):2553.

28. Wolfe F. Editorial: the status of fibromyalgia criteria. Arthritis Rheumatol. 2015;67(2):330-333.

29. Littlejohn G. Fibromyalgia: Honing fibromyalgia diagnosis. Nat Rev Rheumatol. 2014;10(5):267-269.

30. Brummett CM, Urquhart AG, Hassett AL, et al. Characteristics of fibromyalgia independently predict poorer long-term analgesic outcomes following total knee and hip arthroplasty. Arthritis Rheumatol. 2015;67(5):1386-1394.

31. Veldman PH, Reynen HM, Arntz IE, Goris RJ. Signs and symptoms of reflex sympathetic dystrophy: prospective study of 829 patients. Lancet. 1993;342(8878):1012-1016.

32. Schwartzman RJ, McLellan TL. Reflex sympathetic dystrophy. A review. Arch Neurol. 1987;44(5):555-561.

33. Steinbrocker O, Argyros TG. The shoulder-hand syndrome: present status as a diagnostic and therapeutic entity. Med Clin North Am. 1958; 42(6): 1533-1553.

34. Bruehl S, Harden RN, Galer BS, Saltz S, Backonja M, Stanton-Hicks M. Complex regional pain syndrome: are there distinct subtypes and sequential stages of the syndrome? Pain. 2002;95(1-2):119-124. 
35. Harden RN, Bruehl S, Perez RS, et al. Development of a severity score for CRPS. Pain. 2010;151(3):870-876.

36. Littlejohn GO. Reflex sympathetic dystrophy in adolescents: lessons for adults. Arthritis Rheum. 2004;51(2):151-153.

37. Littlejohn G. Therapy: Bisphosphonates for early complex regional pain syndrome. Nat Rev Rheumatol. 2013;9(4):199-200.

38. Littlejohn G. Regional pain syndrome: clinical characteristics, mechanisms and management. Nat Clin Pract Rheumatol. 2007;3(9): 504-511.

39. Littlejohn GO, Guymer EK. Whiplash: same elephant, different room. J Rheumatol. 2014;41(3):411-413.

40. Wolfe F, Clauw DJ, Fitzcharles MA, et al. The American College of Rheumatology preliminary diagnostic criteria for fibromyalgia and measurement of symptom severity. Arthritis Res (Hoboken). 2010; 62(5):600-610.
41. Parkitny L, McAuley JH, Di Pietro F, et al. Inflammation in complex regional pain syndrome: a systematic review and meta-analysis. Neurology. 2013;80(1):106-117.

42. Littlejohn GO, Weinstein C, Helme RD. Increased neurogenic inflammation in fibrositis syndrome. J Rheumatol. 1987;14(5):1022-1025.

43. Oaklander AL, Fields HL. Is reflex sympathetic dystrophy/complex regional pain syndrome type I a small-fiber neuropathy? Ann Neurol. 2009;65(6):629-638.

44. Kosmidis ML, Koutsogeorgopoulou L, Alexopoulos H, et al. Reduction of Intraepidermal Nerve Fiber Density (IENFD) in the skin biopsies of patients with fibromyalgia: a controlled study. J Neurol Sci. 2014; 347(1-2):143-147.

45. Clauw DJ. Fibromyalgia: a clinical review. JAMA. 2014;311(15): $1547-1555$.
Journal of Pain Research

\section{Publish your work in this journal}

The Journal of Pain Research is an international, peer-reviewed, open access, online journal that welcomes laboratory and clinical findings in the fields of pain research and the prevention and management of pain. Original research, reviews, symposium reports, hypothesis formation and commentaries are all considered for publication.

\section{Dovepress}

The manuscript management system is completely online and includes a very quick and fair peer-review system, which is all easy to use. Visit http://www.dovepress.com/testimonials.php to read real quotes from published authors. 